\title{
Erratum: Physical origin of the universal three-body parameter in atomic Efimov physics [Phys. Rev. A 90, 022106 (2014)]
}

\author{
Pascal Naidon, Shimpei Endo, and Masahito Ueda \\ (Received 26 October 2021; published 10 November 2021)
}

DOI: 10.1103/PhysRevA.104.059903

Our original article contains two errors detailed below. None of these errors change the reasonings and conclusions of the article.

First, the black and gray curves shown in Fig. 7 were wrongly computed due to a missing numerical factor in the formulas used to make the calculation. The corrected figure and corresponding caption are shown in the new Fig. 7. As can be seen, the shape of the correct effective three-body potential is slightly different from the published one but remains consistent with the low-energy Faddeev potential. To better approach the limit of deep two-body potentials, the low-energy Faddeev potential shown in the corrected figure is obtained for a two-body interaction that is described by a Lennard-Jones potential with six $s$-wave bound states (the original figure corresponds to three $s$-wave bound states).

Second, the discussion on the hyperangular distribution and associated average hyperangle on p. 4 is based on an erroneous form of this distribution. This discussion is incorrect and should be dismissed as it is not essential to the main discussion of the article. The corresponding part to dismiss starts from "The necessity to deform" and ends with "as well as the couplings to other channels.," including Fig. 4 which should also be dismissed.

In particular, we note that the value of $0.508 \cdots$ given in the published article for the average hyperangle is incorrect. This relatively small value is wrongly interpreted as a manifestation of the elongated geometry of Efimov trimers. A proper calculation using the correct form of the hyperangular distribution gives the value of $\langle\alpha\rangle \approx 0.769$, which turns out to be significantly larger. Although it remains true that Efimov states favor elongated configurations as shown in Fig. 2 of the article, the average value of the hyperangle does not characterize this elongation. Indeed, although elongated configurations in the considered Jacobi set contribute to small values of the hyperangle, elongated configurations in the other two sets contribute to large values.

We thank J. van de Kraats for pointing these errors out and confirming the correct calculations.

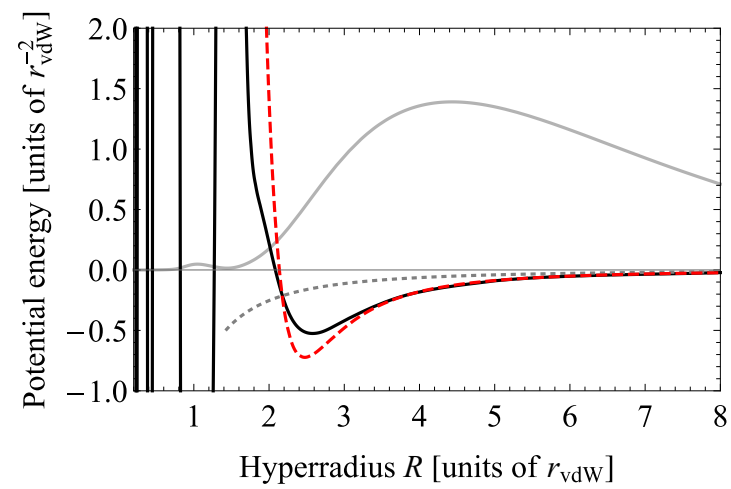

FIG. 7. Three-body probability (solid gray curve in arbitrary units) as a function of the hyperradius and corresponding effective three-body potential (solid black curve), obtained from the separable model Eq. (15). For comparison, the Efimov potential (dotted curve) and the lowenergy Faddeev three-body potential obtained from Eq. (7) for a Lennard-Jones potential with six $s$-wave bound states (red dashed curve) are also represented. Note that for $R<1.5 r_{\mathrm{vdw}}$, the effective potential becomes strongly oscillatory (as can be seen from the nearly vertical lines) in order to reproduce the wave function. Since the physics in that region is correctly described by coupled potentials, the effective potential is not a meaningful construct in that region and the short-range oscillations have no particular significance. 